\title{
Better Visualization of Vermiform Appendix With Tissue Harmonic Imag- ing Compared to Conventional Sonography
}

\author{
Mikail Inal ${ }^{1,}$; Birsen Unal $^{1}$; Yasemin Karadeniz Bilgili ${ }^{1}$ \\ ${ }^{1}$ Department of Radiology, Kirikkale University School of Medicine, Kirikkale, Turkey \\ *Corresponding author: Mikail Inal, Department of Radiology, Kirikkale University School of Medicine, Kirikkale University Campus, Ankara Yolu 7.Km.71450, Yahsihan/Kirikkale, \\ Turkey. Tel:+90-3182252485, E-mail: inal_m@hotmail.com
}

Received: February 9, 2014; Revised: April 16, 2014; Accepted: April 30, 2014

\begin{abstract}
Background: Surgery of appendicitis carries 7-11\% negative appendectomy rates. Sonographically visualized normal appendix precludes unnecessary computed tomography (CT) examination and may reduce negative appendectomy rates. Tissue harmonic imaging (THI) has been reported to improve the overall image quality.

Objective:We aimed to assess whetherTHI is more successful than conventional ultrasonography(US)in detecting normal and pathologic appendices.

Patients and Methods: The study was performed on 185 patients who applied for routine US examinations in whom clinical findings of appendicitis were detected in 25 . We searched for the appendix; applying both THI and conventional US to each patient, one before and the other after the routine US examinations. Patients were divided into two groups; one was evaluated first with conventional US and the other first with THI. When the appendix was found, localization, diameter and time spent for visualization were recorded. Twelve patients were operated; all of whom had appendicitis pathologically. Two methods were compared for: 1 . Success rates in all patients; female, male and child groups separately; 2 . Visualization of pathologic and normal appendices; 3 . Time for visualization of appendix; 4 . Comparison of success rates in the adult and child population. The relationship between the rate of visualization and body mass index was evaluated. Results: The appendix was visualized better by THI in all patients, and in the femaleand male groups $(\mathrm{P}<0.001)$. In children, both methods were more successful compared to adults ( $\mathrm{P}<0.001$, compared to male group, $\mathrm{P}<0.001$, compared to female group), with no difference between the methods $(\mathrm{P}=0.22)$. When only the normal appendices were concerned, there was significant difference between both methods $(\mathrm{P}<0.000)$. Both methods detected pathologic appendices better than normal ones, with a higher ratio for THI $(\mathrm{P}=0.022$ for the THI group, and $\chi^{2}=7.22, \mathrm{P}=0.07$ for the conventional US group). THI visualized the appendix faster. Both methods were more successful in lean patients $(\mathrm{P}=0.004$ for $\mathrm{THI}, \mathrm{P}=0.001$ for conventional US imaging).

Conclusions: THI visualizes appendix better than conventional US. It is a simple and time saving method that may eliminate further diagnostic imaging, and it may decrease negative appendectomy rates and related complications.
\end{abstract}

Keywords:Appendix; Appendicitis; Tissue Harmonic Imaging; Ultrasound Imaging; Diagnosis

\section{Background}

Acute appendicitis is one of the most common diagnoses made in patients with an "acute abdomen." Although the mortality rate has been reduced, a negative appendectomy rate of approximately $5 \%$ to $34 \%$ has remained unchanged when the diagnosis was established on the basis of clinical history, physical exam and laboratory findings (1-5).

Cross-sectional imaging techniques, including ultrasonography (US) US, computed tomography (CT) and more recently magnetic resonance imaging (MRI), have been successfully used to examine patients suspected of appendicitis (1-15). Because of technical improvements, sonography has been reported to reach sensitivities and specificities up to $98 \%$ for the diagnosis of acute appendicitis, a ratio highly dependent on the experience of the sonographer $(5,11-17)$. However, even to experienced sonographers, the normal vermiform appendix is not always visible sonographically. With conventional ultrasound imaging, a normal appendix can be clearly identified in $12-82 \%$ of the cases $(16,18-21)$. This ratio is higher in pathologic appendices (22). Visualization of a normal appearing appendix by cross-sectional imaging techniques in patients suspected of acute appendicitis will prevent negative appendectomy and related complications, not only peroperative, but also late stage complications like chronic right sided lower abdominal pain $(23,24)$.

Harmonic imaging is a technique originally developed in contrast imaging, but now it is widely applied to native tissue imaging, generally called as tissue harmonic imaging (THI). Low amplitude harmonic waveforms are generated by tissue interaction with the ultrasound pulse (25). Preferential display of the harmonic signal can significantly improve image quality, because of a decrease in image degrading echoes from the body wall and those

Copyright ( ) 2014, Tehran University of Medical Sciences and Iranian Society of Radiology; Published by Kowsar. This is an open-access article distributed under the terms of the Creative Commons Attribution-NonCommercial 4.0 International License (http://creativecommons.org/licenses/by-nc/4.0/) which permits copy and redistribute the material just in noncommercial usages, provided the original work is properly cited. 
Inal Met al.

generated by side lobe artifacts. THI has been reported to improve the overall image quality and lesion characterization in abdominal and pelvic imaging (26-28).

\section{Objectives}

We planned this study to determine if THI sonography is superior to conventional sonography in the visualization of vermiform appendix in patients with or without clinical suspicion of acute appendicitis. We focused comparing the efficacy of conventional sonography with THI in detecting the appendix.

\section{Patients and Methods}

The local Ethics Committee of the hospital approved the study, and informed consent was obtained in each case. The study was performed on 185 consecutive patients who had applied for abdominal or pelvic sonographic examination to our department with clinical findings of acute abdomen in 25 of them and other reasons in the remaining ones and who had accepted to be included into the study, Patients who were unwilling to participate or patients with general conditions not suitable for extra ultrasound examination, including emergency room or intensive care unit patients with trauma, severe dyspnea, shock, or patients who needed immediate operation were excluded. Age and gender of the patients were recorded. Body-mass index was calculated in adult patients [weight $(\mathrm{kg}) /$ height $(\mathrm{m})^{2}$ ]. All examinations were performed by the same ultrasound machine with a $5-13 \mathrm{MHz}$ broad band matrix linear transducer (General Electric, model Logic 900, Milwaukee, USA). We used frequencies of 8-10 $\mathrm{MHz}$ for conventional US, and 5 and $10 \mathrm{MHz}$ as transmitted and received frequencies respectively, for THI. Both conventional US and THI methods were performed on each patient, one before and the other after the routine abdominal or pelvic US examinations with an approximate 1 hour interval, by two observers, experienced in abdominal sonographic imaging. Cases with clinical suspicion of appendicitis and sonographically detected appendicitis were examined by the first observer (BU) and the first and second examinations were performed with 15-30 minutes intervals, instead of the 1 hour interval, to prevent any delay in treatment. US evaluation for visualization of the appendix was maximum 5 minutes for each method. If the appendix could not be detected during the 5 minutes of scanning, it was accepted as not detected by that method. We performed the study in two phases. In the first phase, we examined each patient first with THI and second with conventional US. In the second phase of the study, each patient was examined first with conventional US and second with THI.

To find the appendix, we localized the cecum and terminal ileum, then systematically investigated the possible localization of the appendix. We evaluated the right lower quadrant, right upper quadrant, periumbilical area and the pelvis. We observed the appendix as a blind ending tubular structure originating from the cecum. In cases with partially visualized appendices, we always visualized the ileum separately originating from the cecum, confirmed by either observing peristaltism in it or a larger diameter compared to the appendix. If any or both observers thought that they found the appendix, they saved the video images. For those patients, after four examinations (THI and conventional US performed by each of the two observers), the observers come together and examined each saved video image on the machine. If the observers decided that the imaged structure is the appendix, the appendix was accepted as visualized by that method by that observer. If they decided that it was not the appendix, they accepted that the appendix was not visualized. In case of no consensus, the saved images were shared with a third observer and the same protocol was applied. Diameter and localization of the appendix were recorded. Compressible appendices with a diameter smaller than $6 \mathrm{~mm}$ were accepted as normal. Appendices with a larger diameter, distended with fluid and noncompressible in cases with clinical suspicion of appendicitis were accepted as pathologic. The time the appendix was visualized was recorded by the first observer.

After the US examinations, 25 patients with clinical findings of acute abdomen were followed in the hospital and after being discharged from the hospital by phone for two weeks. Surgical and pathological results in operated patients and follow up results in those who did not undergo surgery were obtained and the patients were divided as nonappendicitis and appendicitis groups. All groups were also divided as female, male and children.

The results of THI and conventional US imaging were compared for success rates in all patients, and in the female, male and child groups separately with the McNemar test. 173 patients without clinical findings of acute abdomen who were evaluated with both methods were also tested with McNemar test to assess the power of the method.

The child group was also compared with the adult population for both methods separately with Pearson ChiSquare Test. The effectiveness of both methods between 12 pathologically proven appendicitis and the remaining 173 cases were made by the Pearson Chi-Square test for conventional US, and by Fisher's exact test for THI sonography. The relationship between visualization of the appendix and BMI was searched with Pearson Chi-Square test. The comparison of the time for visualization of the appendix for both methods was made by Paired Samples t-test. A P value less than 0.05 was accepted as statistically significant for all tests.

\section{Results}

The characteristics (age, gender and BMI) of the patients in each group are summarized in Table 1 . When all the patients were concerned, there was no age difference between both groups (for group 1, age: $39.11 \pm 13.97$ years, for 
Inal Met al.

group 2, age: $42.58 \pm 12.62$ years, $\mathrm{P}=0.134)$. There was no statistically significant difference between gender, and BMI of adult patients in both groups ( $\mathrm{p}$ value for gender $=0.393$; regarding BMI, mean BMI for group $1=26.15 \pm 4.96$, mean BMI for group $2=27.8 \pm 4.78$, p value for BMI comparison $=0.051)$. The number of child cases in each group did not show significant difference.

The results of ultrasound evaluations for groups 1 and 2 are summarized in Table 2. Using both imaging methods, the appendix was visualized in 137 (74.05\%) patients by the first observer. THI identified appendix in 136 patients (73.5 $\%)$, conventional US detected appendix in 88 patients ( 47.5 $\%)$. For the second observer, THI identified the appendix in 86 patients ( $49.7 \%$ ), and conventional US detected the appendix in 49 patients $(28.3 \%)$ in the non-appendicitis group. There was significant difference in both methods between the observers (for both methods $\mathrm{P}<0.001$ ). In total, 14 cases (for observer one, six cases and for observer two, eight cases) were evaluated by the third observer, of whom nine were decided as appendix, four cases were accepted as not-appendix and one case was not determined (for observer two) and accepted as non visualized.

When only 173 normal appendices were concerned, there was significant difference between both methods for both observers $(\mathrm{P}<0.000)$. THI sonography significantly better visualized the appendix when all patients were included into statistical evaluation $(\mathrm{P}<0.001)$. Fifty appendices that could not be detected by conventional imaging were clearly visualized by THI (Figure 1). On the other hand, only in two cases in whom the appendix could not be detected with THI, conventional imaging successfully visualized the appendix. The whole length of the appendix was detected in 110 ( $59.4 \%$ ) patients by THI, and in 73 (38.9\%) patients by conventional US by the first observer.

Results of the comparison of the two imaging modalities in patients with and without clinically suspected appendicitis are presented in Table 3. In 160 patients who were evaluated for clinical findings other than acute appendicitis, the first observer visualized the appendix in 114 cases ( $71.5 \%$ ) by THI, and in 70 cases by conventional US $(43,7 \%$ ) (P $<0.001)$. In 25 patients with clinical suspicion of appendicitis, the appendix was visualized by THI in $22(88.8 \%)$ and by conventional US imaging in 18 (72\%). One of the patients in this group in whom the appendix could not be detected underwent CT imaging, and a normal appendix was seen. The remainder of this group did not get further diagnostic imaging. Twelve patients underwent surgery, and the rest were followed up clinically. All patients were discharged from the hospital within one week. In all patients who underwent surgery, surgical and pathologic findings were positive for appendicitis. In these cases, the appendix had been visualized by THI in all cases and by conventional US imaging in $75 \%$ of the cases (Figure 2). Both methods detected pathologic appendices more successfully than normal ones ( $\mathrm{P}=0.022$ for THI group, and $\chi^{2}=7.22, \mathrm{P}=0.07$ for conventional US group). THI detected three patients who were overlooked by conventional imaging. Because of the small number of patients with appendicitis, statistical evaluation could not be performed for this group.

In children under 16 years of age, US imaging, either conventional or THI, was successful in visualizing the appendix with no significant difference between the methods ( $\mathrm{P}$ $=0.22$ ). Compared to female and male groups, both methods were significantly more successful in children $\left(\chi^{2}=\right.$ 41.7, $\mathrm{P}<0.001$ compared to the female group, and $\chi^{2}=12.57$, $\mathrm{P}<0.001$ compared to the male group). THI was superior to conventional US in adult patients $(\mathrm{P}=0.001$ for the male group, and $\mathrm{P}<0.001$ for the female group).

There was a significant correlation between BMI and visualization of the appendix. The ratio of visualization in patients with a BMI lower than 25 , was $80.4 \%$ by THI and $46.4 \%$ by conventional imaging. In patients with a higher BMI, these ratios were $56.4 \%$ and $20.5 \%$ for THI and conventional imaging, respectively $\left(\chi^{2}=8.31\right.$ and $\mathrm{P}=0.004$ for THI, $\chi^{2}=10.11$ and $\mathrm{P}=0.001$ for conventional US imaging).

The mean examination time for THI was $94 \pm 68$ seconds, and it was $122 \pm 84$ seconds for conventional US. Examination time for the two techniques in the appendix-visualized cases was significantly different $(\mathrm{P}=0.021)$.

The most common localization of the appendix was the right lower quadrant, anterior to iliac vessels. Retrocecal appendix was found in 16 patients, of whom nine were children. In three patients, the appendix was detected in the right paracolic gutter, in one patient, it was located in the subhepatic region. Three appendices were mobile, changing position with compression and time, moving to the right and left of the cecum. Two appendices extended to the umbilicus.

The diameter of the appendix was significantly larger in the appendicitis group compared to the non-appendicitis group $(\mathrm{Z}=-5.67, \mathrm{P}<0.001)$. In all 12 patients with appendicitis, the diameter of the appendix was larger than $6 \mathrm{~mm}$ (Table 4). In only two of the remaining 173 patients, the diameter was larger than $6 \mathrm{~mm}$, the diameter was $7.2 \mathrm{~mm}$ and confined to the distal one-third part of the appendix in one case, and $6.9 \mathrm{~mm}$ and covering the whole length in the other. So the sensitivity, specificity, positive and negative predictive values for the diameter over $6 \mathrm{~mm}$ was determined as $85.7 \%, 100 \%, 100 \%$ and $98.8 \%$, respectively.

\begin{tabular}{|c|c|c|c|}
\hline & No. & Age, $y$, Mean \pm SD & BMI, Mean \pm SD $^{b}$ \\
\hline \multicolumn{4}{|l|}{ Group 1} \\
\hline Female & 46 & $38.7 \pm 14.8$ & $26.7 \pm 5.76$ \\
\hline Male & 18 & $40.9 \pm 12.4$ & $25.5 \pm 3.4$ \\
\hline Child & 26 & $7.8 \pm 3.9$ & - \\
\hline \multicolumn{4}{|l|}{ Group 2} \\
\hline Female & 47 & $42.3 \pm 10.1$ & $28.9 \pm 5.1$ \\
\hline Male & 23 & $44.9 \pm 12.9$ & $25.8 \pm 5.4$ \\
\hline Child & 25 & $8.7 \pm 4.1$ & - \\
\hline
\end{tabular}

${ }^{a}$ Group 1: patients imaged first with conventional US, second with tissue harmonic imaging, Group 2: patients imaged first with tissue harmonic imaging, second with conventional US.

b Abbreviations: BMI, body mass index; SD, standard deviation. 
Inal Met al.

\section{Discussion}

To the best of our knowledge, there is no study comparing conventional US and THI in appendix detection. The diagnosis of acute appendicitis, established on the basis of clinical history, physical and laboratory findings results in an overall accuracy of approximately $80 \%$, with a negative appendectomy rate of approximately $20 \%$. Investigators in prior studies have reported that negative appendectomy rates varies by patient gender, with a range of $5-16 \%$ in men and $11-34 \%$ in women (1-5). These gender-based differences reflect the fact that the diagnosis of appendicitis on clinical bases alone may be extremely difficult in female patients because of the broad overlap of symptoms of acute gynecologic abnormalities. The recent reports reveal that with the advent of CT, US and MRI, the accuracy and normal appendix
Table 2. Visualization of the Appendix in Both Groups for Observer One ${ }^{\text {a }}$

\begin{tabular}{lccc}
\hline & \multicolumn{3}{c}{ Visualization of Appendix } \\
\cline { 2 - 4 } & No. & \multicolumn{2}{c}{ First and Second Examinations } \\
\cline { 2 - 4 } & & THI & CON \\
\hline Group 1 & & \\
Female & 46 & 32 & 16 \\
Male & 18 & 11 & 8 \\
\hline Child & 26 & 25 & 20 \\
Group 2 & & & \\
Female & 47 & 31 & 15 \\
Male & 23 & 15 & 6 \\
\hline Child & 25 & 22 & 23 \\
\hline
\end{tabular}

a Group 1: patients imaged first with conventional US, second with THI. Group 2: patients imaged first with THI, second with conventional US. CON: conventional US, THI: tissue harmonic US

Table 3. The Number and Percentages of Visualized Appendices by THI and Conventional US by Both Observers ${ }^{a}$

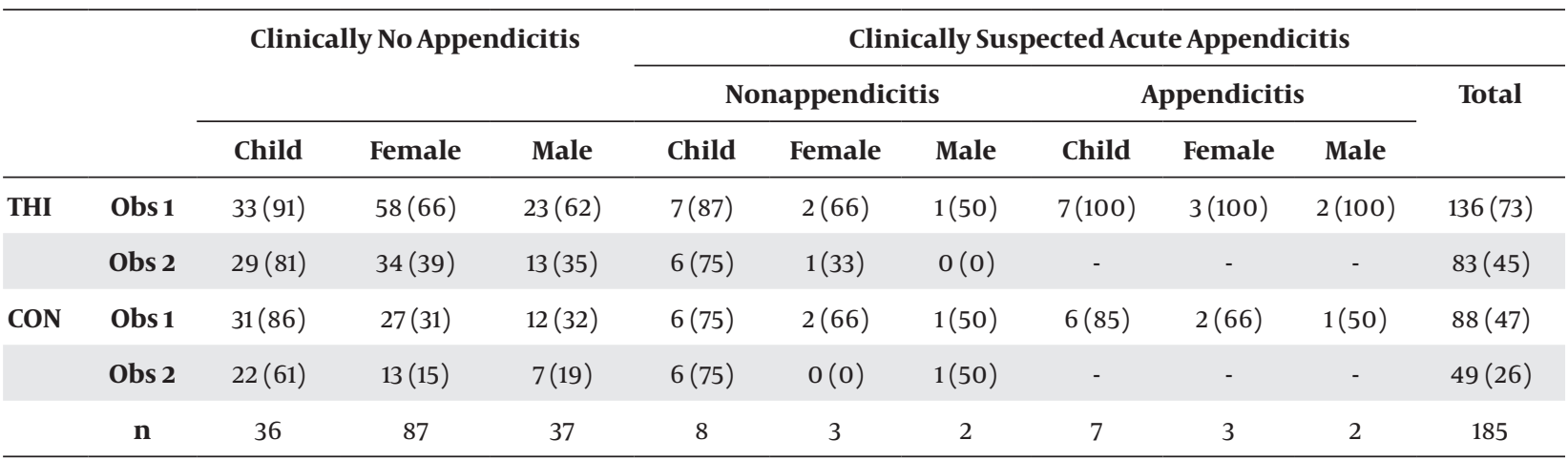

a THI: tissue harmonic imaging. CON: conventional ultrasound imaging. n: total number of patients in each group. Obs: observer. Numbers in parentheses are the percentages

Table 4 . Diameter of Appendix in Groups ${ }^{\text {a }}$

\begin{tabular}{lccccc}
\hline & Child & Female & Male & Total & Minimum-Maximum \\
\hline Non Appendicitis & $0.40 \pm 0.08$ & $0.39 \pm 0.10$ & $0.40 \pm 0.11$ & $0.39 \pm 0.09$ & $0.24-0.72{ }^{\mathrm{b}}$ \\
Appendicitis & $0.82 \pm 0.15$ & $0.81 \pm 0.20$ & $0.84 \pm 0.9$ & $0.81 \pm 0.15$ & $0.69-1.11^{\mathrm{b}}$ \\
\hline
\end{tabular}

a diameter values are given as mean \pm standard deviation in $\mathrm{cm}$.

$\mathrm{b}$ appendix diameters were significantly different between appendicitis and nonappendicitis groups $(\mathrm{P}<0.05)$.
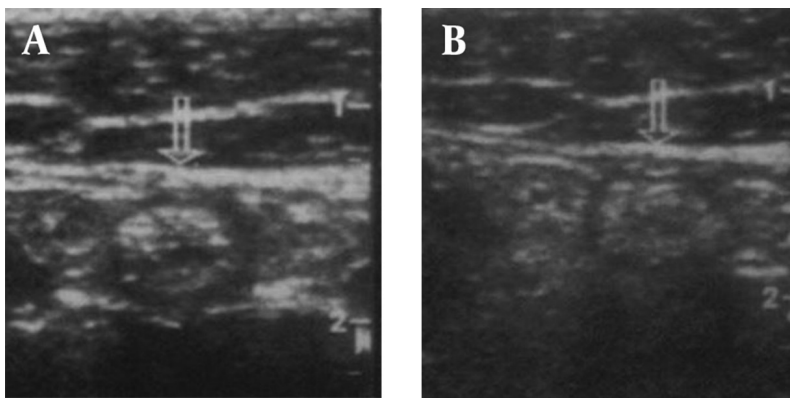

Figure 1. THI (A) and conventional US (B) images of a normal appendix showing a slightly better image quality of THI
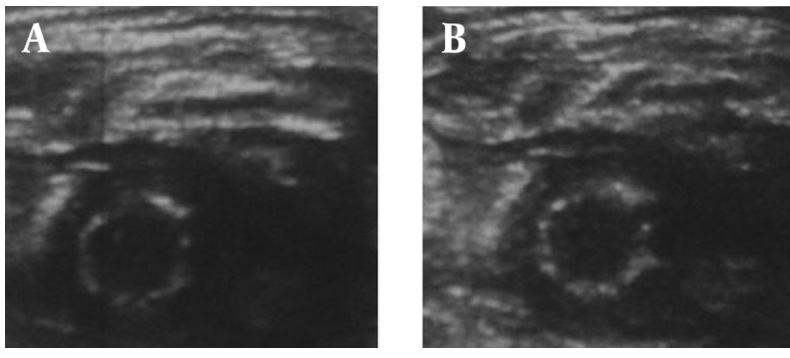

Figure 2. THI(A) and conventional US (B) images of a pathologic appendix showing adequate image quality for both images 
Inal Met al.

removal improved significantly, particularly in those patients with atypical symptoms. It has also been reported thatpatients who benefit most from the preoperative imaging are women. With CT and US imaging, negative appendectomy rates decreased to $7-11 \%$ from $28-34 \%$ in this patient population $(1,4)$. In general, CT has been accepted superior to US in the diagnosis of appendicitis, with higher sensitivity, specificity, accuracy and lower normal appendix removal. The sensitivity, specificity and accuracy of CT imaging were reported as $93-100 \%, 85-99 \%$, and $94-97.6 \%$, respectively, with higher ratios in men compared to women (5-10). The corresponding values for US imaging shows a wide range; 50-99.3\%, 68.1-98\% and 83$98 \%$, respectively, with higher ratios in examinations performed by highly qualified sonographers $(5,11-15)$. These ratios were higher when only the visible appendices were included into statistical evaluations (16). Visualization of the appendix depends on not only the experience of the observer but also patient-related factors such as obesity, bowel gas, atypical position of the cecum and the retrocecal position of the appendix $(13,14,17)$. To improve the visualization of the appendix, hydrocolonic US, a method applied with saline enema has been used. This technique increased the sensitivity of US imaging from 50\% to $75 \%$ (5). The posterior manual compression technique is another method that has increased the ratio of appendix visualization from $85 \%$ to $95 \%$ (18).

The normal appendix can be visualized in approximately $12-82 \%$ of patients $(16,18-21)$. In inflamed appendices, this ratio increases up to $95 \%$ (22). On the other hand, acute appendicitis can be found in a remarkable number of patients with nonvisualized appendices (16).

Visualization of the normal appendix is important in preventing normal appendix removal and related preoperative and postoperative complications being most commonly infections and chronic right lower quadrant pain $(23,24)$. Finding a normal appendix is strongly against the decision of performing surgery in patients with positive clinical findings in the absence of other surgical conditions.

THI is a sonographic technique that can potentially provide higher quality images compared to conventional sonographic techniques (25). Harmonics are frequencies generated by propagation of the ultrasound beam through tissue that occur at multiples of the fundamental or transmitted sonographic frequency. THI sonography uses these harmonic frequencies to produce the sonogram, instead of using the frequency spectrum that is transmitted to the patient as in conventional US (25). Imaging using harmonic frequencies offers several potential advantages, including improved lateral resolution, and reduced side-lobe artifacts. Increased lateral resolution improves the ability to resolve small anatomic structures and detail. Reduction in side-lobe artifacts improves the signal-to-noise ratio, resulting in an image in which tissue appears brighter and cavities appear darker. The harmonic signal is generated within the tissue; there- fore, artifacts from the body wall may be less pronounced with THI. In general, the studies performed for evaluation of abdominal pathologies, comparing THI with conventional US revealed improved image quality, lesion detection and characterization (particularly fluid-solid differentiation) with $\mathrm{THI}(26,27)$. In a study performed by Hann et al. on hepatic lesions, THI had a significant impact on clinical decision-making in $10 \%$ of the patients mostly due to detection of additional lesions or differentiation of small cystic lesions from solid masses. They observed an improvement for both near and far-field image quality with THI (26). Shapiro et al. similarly stated that THI penetrated better than conventional US in imaging for pancreas pathologies (27). Oktar et al. concluded that THI was significantly superior for revealing stone diseases, liver cysts, gallbladder polyps, and uterine myomas and overall image quality, lesion conspicuity, and elimination of artifacts (28).

We performed this study to determine if the theoretic advantages of THI sonography resulted in improvement in the detection of appendix. In the adult population, we observed a significantly higher ratio for visualization of the appendix with THI sonography. Fifty appendices detected by THI, could not be seen with conventional imaging. In all these 50 patients, we observed that far-field image quality was superior with THI sonography, a finding supporting the results of Hann et al. and Shaphiro et al. Even though the distance between the appendix and skin is longer in adult patients and penetration of THI is shorter, THI is better in visualizing the appendix $(26,27)$. The success rate of THI in pathologically proven appendicitis was also higher. All patients with appendicitis were detected by THI, but only $75 \%$ were detected by conventional US imaging. Time for visualization was also shorter for THI sonography.

Using two methods together, $74.05 \%$ of all appendices were visualized. We thought that the reason for unsuccessful examinations were mostly due to the retrocecal position of the appendix, because we detected retrocecal appendix in 16 patients with a percentage of $0.8 \%$, which was lower than surgically determined ratios. Grunditz et al. found retrocecal appendix in $17 \%$ of the operated cases in their series, which consisted of 247 patients (29). THI could visualize the whole lengthwise of the appendix more successfully than conventional US, and this may be important in the diagnosis of cases with focal appendicitis. In adult patients, we found a significant correlation between appendix visualization and BMI, which was valid for both imaging methods and was consistent with the results of a study conducted by Josephson et al.(30).

Technical aspects affecting the ability of the sonographer to achieve adequate compression of the RLQ such as obesity, severe pain or abdominal guarding, excessive bowel gas, and an uncooperative patient can be listed as limitations of the study. In addition, patients who are unwilling to participate or patients with an unsuitable general condition for extra ultrasound examination, 
such as emergency room or intensive care unit patients with trauma, severe dyspnea, shock, or patients who need immediate operation have not been included in the present study and these are the main limitations of the study. Since we included consecutive patients, the number of cases with appendicitis was low, which was also a limitation. Another limitation was the low number of male patients compared to female patients, which was due to pelvic examinations that were mostly required for female patients because of more pelvic and gynecological complaints. Lack of assessment of intraobserver reliability and performing the study in two phases instead of block randomization were other technical limitations. We conclude that THI imaging better visualizes the appendix in adult and child patients. It is a simple, time and cost effective method that we believe will reach the success rates of CT and will eliminate the need for further diagnostic imaging. It may also help to prevent negative appendectomy rates and provide cost saving for the hospital.

\section{Acknowledgements}

The author acknowledges the help of Mehmet Ekici from the Department of Pulmonary Disease for statistical analysis.

\section{References}

1. Bendeck SE, Nino-Murcia M, Berry GJ, Jeffrey RB, Jr.. Imaging for suspected appendicitis: negative appendectomy and perforation rates. Radiology. 2002;225(1):131-6.

2. Andersson RE, Hugander A, Thulin AJ. Diagnostic accuracy and perforation rate in appendicitis: association with age and sex of the patient and with appendicectomy rate. Eur J Surg. 1992;158(1):37-41.

3. Velanovich V, Harkabus MA, Tapia FV, Gusz JR, Vallance SR. When it's not appendicitis. Am Surg. 1998;64(1):7-11.

4. Rao PM, Rhea JT, Rattner DW, Venus LG, Novelline RA. Introduction of appendiceal CT: impact on negative appendectomy and appendiceal perforation rates. Ann Surg. 1999;229(3):344-9.

5. Kan JH, Fines BP, Funaki B. Conventional and hydrocolonic US of the appendix with CT correlation performed by on-call radiology residents. Acad Radiol. 2001;8(12):1208-14.

6. Lane MJ, Liu DM, Huynh MD, Jeffrey RB, Jr., Mindelzun RE, Katz DS. Suspected acute appendicitis: nonenhanced helical CT in 300 consecutive patients. Radiology. 1999;213(2):341-6.

7. Yetkin G, Basak M, Isgor A, Kebudi A, Akgun I. Can negative appendectomy rate be decreased by using spiral computed tomography without contrast material? Acta Chir Belg. 2002;102(5):334-7.

8. Raman SS, Lu DS, Kadell BM, Vodopich DJ, Sayre J, Cryer H. Accuracy of nonfocused helical CT for the diagnosis of acute appendicitis: a 5-year review. AJR Am J Roentgenol. 2002;178(6):1319-25.

9. Balthazar EJ, Birnbaum BA, Yee J, Megibow AJ, Roshkow J, Gray C. Acute appendicitis: CT and US correlation in 100 patients. Radiology. 1994;190(1):31-5.

10. Horton MD, Counter SF, Florence MG, Hart MJ. A prospective trial of computed tomography and ultrasonography for diagnosing appendicitis in the atypical patient. Am J Surg. 2000;179(5):379-81.

11. Fujii Y, Hata J, Futagami K, Hamada T, Mitsuoka H, Teramen K, et al. Ultrasonography improves diagnostic accuracy of acute appendicitis and provides cost savings to hospitals in Japan.J Ultrasound Med. 2000;19(6):409-14.

12. Chen SC, Chen KM, Wang SM, Chang KJ. Abdominal sonography screening of clinically diagnosed or suspected appendicitis before surgery. World J Surg. 1998;22(5):449-52.

13. Uebel P, Weiss H, Trimborn CP, Fiedler L, Bersch W. [Ultrasound diagnosis of acute appendicitis-possibilities and limits of the method-results of prospective and retrospective clinical studies]. Ultraschall Med.1996;17(3):100-5.

14. Schwerk WB. [Ultrasound first in acute appendix? Unnecessary laparotomies can often be avoided]. MMW Fortschr Med. 2000;142(26-27):29-32.

15. Rettenbacher T, Hollerweger A, Gritzmann N, Gotwald $T$ Schwamberger K, Ulmer H, et al. Appendicitis: should diagnostic imaging be performed if the clinical presentation is highly suggestive of the disease? Gastroenterology. 2002;123(4):992-8.

16. Himeno S, Yasuda S, Oida Y, Mukoyama S, Nishi T, Mukai M, et al. Ultrasonography for the diagnosis of acute appendicitis. Tokai J Exp Clin Med. 2003;28(1):39-44.

17. Rettenbacher T, Hollerweger A, Macheiner P, Gritzmann N. [Ultrasonography of the normal vermiform appendix]. Ultraschal Med.1997;18(3):139-42.

18. Lee JH, Jeong YK, Hwang JC, Ham SY, Yang SO. Graded compression sonography with adjuvant use of a posterior manual compression technique in the sonographic diagnosis of acute appendicitis. AJR Am J Roentgenol. 2002;178(4):863-8.

19. Lehmann D, Uebel P, Weiss H, Fiedler L, Bersch W. [Sonographic representation of the normal and acute inflamed appendix-in patients wi right-sided abdominal pain]. Ultraschall Med. 2000;21(3):101-6.

20. Rioux M. Sonographic detection of the normal and abnormal appendix. AJR Am J Roentgenol.1992;158(4):773-8.

21. Tarantino L, Giorgio A, de Stefano G, Scala V, Esposito F, Liorre G et al. Acute appendicitis mimicking infectious enteritis: diagnostic value of sonography.J Ultrasound Med. 2003;22(9):945-50.

22. Simonovsky V. [Detection of acute appendicitis using ultrasonography]. Rozhl Chir. 2000;79(5):215-20.

23. Gough IR, Morris MI, Pertnikovs EI, Murray MR, Smith MB, Bestmann MS. Consequences of removal of a "normal" appendix. Med J Aust. 1983;1(8):370-2.

24. Walker SJ, West CR, Colmer MR. Acute appendicitis: does removal of a normal appendix matter, what is the value of diagnostic accuracy and is surgical delay important? Ann R Coll Surg Engl. 1995;77(5):358-63.

25. Ward B, Baker AC, Humphrey VF. Nonlinear propagation applied to the improvement of resolution in diagnostic medical ultrasound. J Acoust Soc Am. 1997;101(1):143-54.

26. Hann LE, Bach AM, Cramer LD, Siegel D, Yoo HH, Garcia R. Hepatic sonography: comparison of tissue harmonic and standard sonography techniques. AJR Am J Roentgenol. 1999;173(1):201-6.

27. Shapiro RS, Wagreich J, Parsons RB, Stancato-Pasik A, Yeh HC, Lao $\mathrm{R}$. Tissue harmonic imaging sonography: evaluation of image quality compared with conventional sonography. AJR Am J Roentgenol.1998;171(5):1203-6.

28. Oktar SO, Yucel C, Ozdemir H, Uluturk A, Isik S. Comparison of conventional sonography, real-time compound sonography, tissue harmonic sonography, and tissue harmonic compound sonography of abdominal and pelvic lesions. AJR Am J Roentgenol. 2003;181(5):1341-7.

29. Grunditz T, Ryden CI, Janzon L. Does the retrocecal position influence the course of acute appendicitis. Acta Chir Scand. 1983;149(7):707-10.

30. Josephson T, Styrud J, Eriksson S. Ultrasonography in acute appendicitis. Body mass index as selection factor for US examination. Acta Radiol. 2000;41(5):486-8. 